Sensors 2015, 15, 16040-16059; doi:10.3390/s150716040

ISSN 1424-8220

www.mdpi.com/journal/sensors

Article

\title{
Traffic Behavior Recognition Using the Pachinko Allocation Model
}

\author{
Thien Huynh-The ${ }^{1}$, Oresti Banos ${ }^{1}$, Ba-Vui Le ${ }^{1}$, Dinh-Mao Bui ${ }^{1}$, Yongik Yoon ${ }^{2}$ and \\ Sungyoung Lee ${ }^{1, *}$ \\ ${ }^{1}$ Department of Computer Engineering, Kyung Hee University, Suwon 446-701, Korea; \\ E-Mails: thienht@oslab.khu.ac.kr (T.H.-T.); oresti@oslab.khu.ac.kr (O.B.); \\ lebavui@oslab.khu.ac.kr (B.-V.L.); mao.bui@khu.ac.kr (D.-M.B.) \\ ${ }^{2}$ Department of Multimedia Science, Sookmyung's Women University, Seoul 140-742, Korea; \\ E-Mail: yiyoon@sookmyung.ac.kr \\ * Author to whom correspondence should be addressed; E-Mail: sylee@ oslab.khu.ac.kr; \\ Tel.: +82-31-201-2514.
}

Academic Editor: Jesús Fontecha

Received: 14 April 2015 / Accepted: 1 July 2015 / Published: 3 July 2015

\begin{abstract}
CCTV-based behavior recognition systems have gained considerable attention in recent years in the transportation surveillance domain for identifying unusual patterns, such as traffic jams, accidents, dangerous driving and other abnormal behaviors. In this paper, a novel approach for traffic behavior modeling is presented for video-based road surveillance. The proposed system combines the pachinko allocation model (PAM) and support vector machine (SVM) for a hierarchical representation and identification of traffic behavior. A background subtraction technique using Gaussian mixture models (GMMs) and an object tracking mechanism based on Kalman filters are utilized to firstly construct the object trajectories. Then, the sparse features comprising the locations and directions of the moving objects are modeled by PAM into traffic topics, namely activities and behaviors. As a key innovation, PAM captures not only the correlation among the activities, but also among the behaviors based on the arbitrary directed acyclic graph (DAG). The SVM classifier is then utilized on top to train and recognize the traffic activity and behavior. The proposed model shows more flexibility and greater expressive power than the commonly-used latent Dirichlet allocation (LDA) approach, leading to a higher recognition accuracy in the behavior classification.
\end{abstract}


Keywords: traffic behavior modeling; closed-circuit television (CCTV) system; pachinko allocation model; video-based road surveillance

\section{Introduction}

Human behavior analysis (HBA), an integral component of many video surveillance systems, is a research area that has recently attracting attention from the computer vision and artificial intelligence communities. The aim of visual surveillance is to detect, recognize and track moving objects from image sequences and to further understand and describe object behaviors. Visual surveillance in dynamic scenes has been considered in a wide range of potential applications [1], such as security guard services in smart buildings [2-4], traffic surveillance in urban areas [5,6] and access control in specific places [7]. In surveillance applications involving people or vehicles, the behaviors can be analyzed based on the human postures [8-11], the object trajectories [12,13] and the tracking information [14]. This information can be combined to recognize more complex contexts, such as vehicle interactions $[15,16]$, human interactions [17,18] and human to vehicle interactions [19]. Given the large amount of surveillance video data available from closed-circuit television (CCTV) systems and the real-time nature of surveillance applications, it is desirable to provide an automatic operating system that may reduce human intervention as much as possible.

One of the most important applications of surveillance systems, automatic road surveillance, has received increasing interest in recent years. In this domain, the learning of the traffic behavior appears to be the most complex task, especially in highly dynamic environments [5]. A behavior is basically represented through the combination of atomic activities, which are modeled from object features, such as location, direction or tracking information, among others. In order to learn the behavior, the correlation between potential features in the spatial and temporal dimension is normally used as part of probabilistic graphical models [16]. The most widely-used probabilistic approach is the hidden Markov models (HMMs), in which the unknown behaviors are derived from the combination of sequential states with a given likelihood. Although HMM is a simple and efficient model for sequential state estimation, its performance in terms of recognition accuracy tends to degrade in the case of complex scenarios, including multiple objects and long-term temporal behaviors [14]. This limitation has motivated the recent use of topic models as a more effective solution.

Compared with previous works, the authors propose a method for traffic behavior learning for multi-object environments. Firstly, the feature-book, including object trajectories, is created from sparse tracking information in the temporal-spatial dimension. The foreground containing moving objects is extracted using a background subtraction technique based on the Gaussian mixture models (GMM). The Kalman filter is then utilized to track the trajectories of the detected objects in frame sequences. Traffic activities and behaviors are then generated from the identified trajectories with a flexible topic model, namely the pachinko allocation model (PAM). PAM provides a full correlation between features and activity and activities and behavior based on an arbitrary directed acyclic graph (DAG) structure. Finally, a multi-class support vector machine (SVM) technique is employed to classify the activity and behavior according to the outputs of the PAM model. The remainder of this paper is organized as 
follows. Section 2 provides the discussion of related works. Section 3 describes the proposed method for modeling and identification of traffic behavior. The experimental setup, results and discussion are presented in Section 4. Finally, the conclusions of this paper are summarized in Section 5.

\section{Related Work}

Diverse contributions have been made in the development of behavior recognizers for smart building surveillance applications. The switching hidden semi-Markov model (S-HSMM), an extended version of HMMs, was first introduced by Duong et al. [12] for learning and recognizing daily living human activities. The activities are modeled in two stages-presentation and duration-using HSMMs in the bottom layer and the presentation of the sequence of atomic activities in the top layer. To detect abnormal behaviors in indoor environments, a sparse reconstruction analysis of movement trajectories was proposed by Li et al. [13]. Although this approach is efficient for small training sets, its performance is sensitive to the numbers of control points used in the cubic B-spline curve approximation, especially for large training sets. Xiang et al. [20] proposed a novel framework developed for automatic behavior profiling and online abnormalcy detection using a dynamic Bayesian network (DBN). In this work, the behavior patterns are grouped by using the relevant eigenvectors of the normalized affinity matrix. Unlike some existing techniques, the present approach is apparently simple and robust, even with outliers in the input data. A two-stage learning algorithm based on the time-delayed probabilistic graphical model (TD-PGM) was formulated by Loy et al. [21] to effectively detect and localize unusual global events as context-incoherent patterns. Unlike other approaches, the proposed scheme in [22] detects multi-camera group activities from intra- and inter-cameras without a topology inference. The context is characterized by the structure of the hidden variables, which are developed from a discriminative graphical model (DGM). However, it has the drawback of the co-occurrence relationship being captured among activity patterns, which cannot be completely obtained. Rather than propose a novel learning model, Huang et al. [23] focused on improving the positioning accuracy by combining the head location and posture recognition as a multi-camera positioning algorithm.

In automatic road surveillance, the vehicle activities and behaviors are detected and recognized for monitoring and warning purposes. A simple method of robustly detecting moving objects was suggested by Kermani et al. [6] for recognition of abnormalities in both indoor and outdoor scenarios. The Bayesian change detection (BCD) algorithm is used to eliminate noise, shadows, illumination variations and repeated motions on the background. To produce an analysis of the behavior of moving objects, a generic framework [24] was constructed with two modular blocks: the first is moving region detection and tracking; the second is the integration of the trajectory and geospatial context. A combination of Bayesian computer vision system (BCVS) and coupled hidden Markov models (CHMMs) [14] was suggested for modeling of pedestrian interactions in outdoor environments. In another study, DBN was used for behavior recognition in a road detection system (BRRD) [25] through vehicle sensor networks (VSNs) to infer road events. Moreover, group detection using collaborative filtering provides an improvement in detection performance. HMMs were also applied by Brand et al. [15] to organize observed activities based on minimizing the entropy of component distributions for both office activities and outdoor traffic flows. This framework was further adapted to infer the hidden state from 
ambiguous videos by referencing human body orientations and poses. In [5], Xiang et al. recommended a dynamically multi-linked hidden Markov model (DML-HMM) comprising expectation-maximization (EM) clustering and the Bayesian information criterion (BIC) classification. Moreover, dynamic probabilistic networks (DPNs) have been formulated to model the temporal and causal correlations among discrete events for holistic scene-level behavior interpretation. To unify simple and complex action recognition, Sanroma et al. [26] encoded simple action HMMs within a stochastic grammar that models complex actions. This approach substantially improved the classification accuracy by developing the higher activity layers based on the recognition of simple actions. Another framework using an extension of stochastic context-free grammar (SCFG) to model the complex temporal relations between atomic activities was suggested by Zhang et al. [27]. Their main innovation was a multithread parsing algorithm adopted for the trained event rule induction for recognition instead of the common parser. The common limitation of most of these HMM-based approaches refers to the need of large amounts of training data, since they do not scale well for complex behavior cases.

The use of topic models for context learning has recently been introduced. Zhao et al. [16] suggested an effective framework comprised of three steps: construct the motion frame codebook, compose the atomic activities through the LDA-based topic model and classify the behavior with the rough set scheme. Detecting and recognizing urban activity using topic models from online geo-location data was proposed by Hasan et al. [28]. Two well-known topic modeling algorithms, the latent Dirichlet allocation (LDA) and hierarchical Dirichlet process (HDP), have been typically employed in HBA systems. The delta-dual hierarchical Dirichlet process (dDHDP), which is an extension of HDP, was designed by Haines et al. [29] for jointly learning both normal and abnormal behavior using weakly supervised training examples. A new topic model is introduced by Hospedales et al. [30] to overcome the drawbacks on the sensitivity, robustness and efficiency of object behavior mining. The topic model, namely the Markov clustering topic model (MCTM), builds on existing dynamic Bayesian network models and Bayesian topic models. This model was demonstrated to succeed on the unsupervised mining of behaviors in complex and crowded public scenes. Three hierarchical Bayesian models [31] — the LDA mixture model, the HDP mixture model and the dual-HDP model-were proposed in Wang's research. These models cluster both motion pixels and video clips into atomic activities and into interactions. The atomic activities are modeled as distributions over low-level visual features, such as the location and direction of motion pixels, while multi-agent interactions are modeled as distributions over atomic activities. Although many challenging visual surveillance tasks have been completed in the above research, the task of considering activities and interactions with complicated temporal structures remains.

\section{Methodology}

The proposed method consists of the following modules: feature extraction, topic modeling and classification, as presented in Figure 1. 


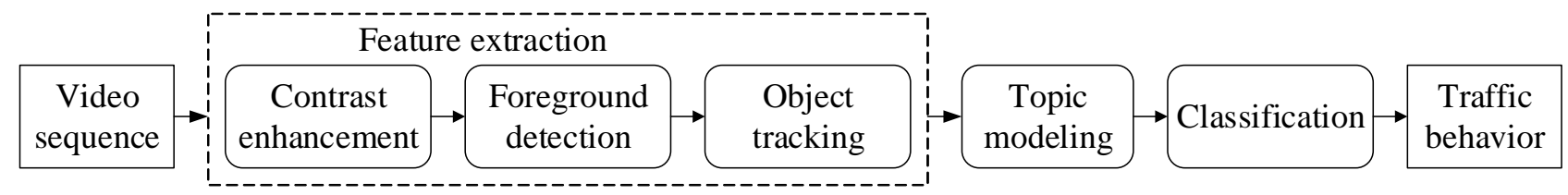

Figure 1. Proposed traffic behavior recognition workflow.

\subsection{Feature Extraction}

As a preprocess for improving the quality of input video sequences, an efficient histogram equalization [32] is used to enhance the overall contrast. The object trajectories in the input video captured from the CCTV system are then extracted using a combined background subtraction and tracking technique. The adaptive-K Gaussian mixture model (AK-GMM) [33] is used to establish the model for background estimation on account of its robustness under changing environments. The moving objects are distinguished from the foreground using a background subtraction technique [34]. As a result, the object coordinates are obtained as the centroid point of the bounding box surrounding the potential object. The Kalman filter is used for tracking objects, and it enables the prediction of an object's future location, a reduction of noise introduced by inaccurate detections and facilitation of the association of multiple objects to their tracks.

The object trajectories are represented in the temporal-spatial dimension. Example object trajectories illustrated in the spatial domain are shown in Figure 2a; those in the temporal-spatial domain are shown in Figure 2b. To determine the orientation of the object trajectory, the absolute angle $\alpha$ of the current location is calculated through the following equation:

$$
\alpha_{i}=\arcsin \left(\frac{y_{i}}{\sqrt{x_{i}^{2}+y_{i}^{2}}}\right)
$$

where $\left(x_{i}, y_{i}\right)$ are the coordinates of the object at the $i$-th frame. A direction computation example is shown in Figure 2c. Only one angle value corresponding to the current frame is acquired. Each moving object is described by two features: the location and the direction. During a specific time period of the input video, which is presented under the number of input frames from $t_{a}$ to $t_{b}$, the trajectory of an object is formed as:

$$
\mathcal{O}_{k}^{t_{a-b}}=\left[\left(x_{k}^{t_{a}}, y_{k}^{t_{a}}, \alpha_{k}^{t_{a}}\right),\left(x_{k}^{t_{a}+1}, y_{k}^{t_{a}+1}, \alpha_{k}^{t_{a}+1}\right), \ldots,\left(x_{k}^{t_{b}}, y_{k}^{t_{b}}, \alpha_{k}^{t_{b}}\right)\right]
$$

where $x_{k}^{t_{a}}$ and $y_{k}^{t_{a}}$ are the $X$ and $Y$ coordinate, respectively. $\alpha_{k}^{t_{a}}$ is the moving direction of the $k$-th detected object at current frame $t_{a}$. The object $O_{k}$ presents the trajectory vector in $\left(t_{b}-t_{a}\right)$ frames. Assuming that each input video has $n$ frames, the trajectory is defined as follows:

$$
\mathcal{O}_{k}^{n}=\left[\left(x_{k}^{1}, y_{k}^{1}, \alpha_{k}^{1}\right),\left(x_{k}^{2}, y_{k}^{2}, \alpha_{k}^{2}\right), \ldots,\left(x_{k}^{n}, y_{k}^{n}, \alpha_{k}^{n}\right)\right]
$$




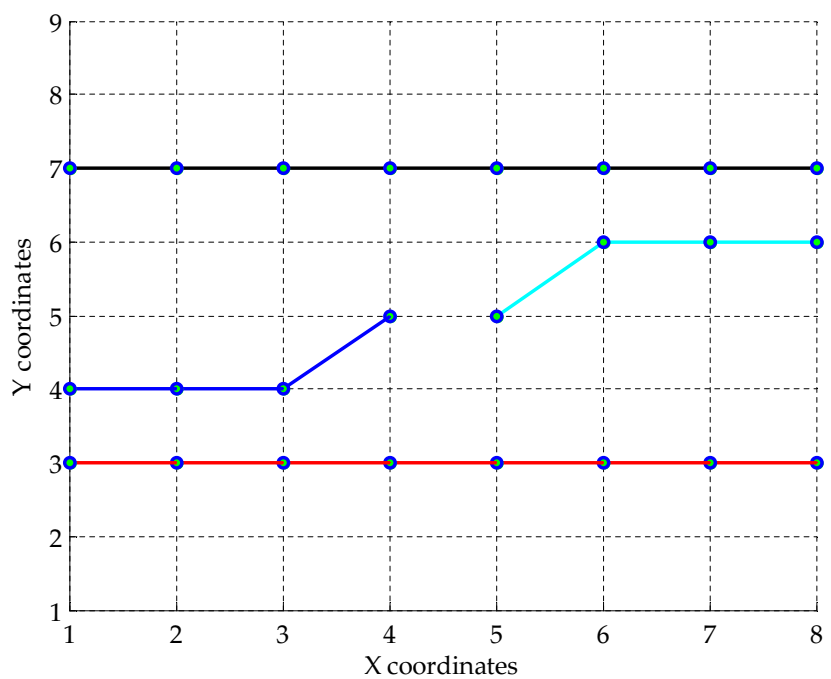

(a)

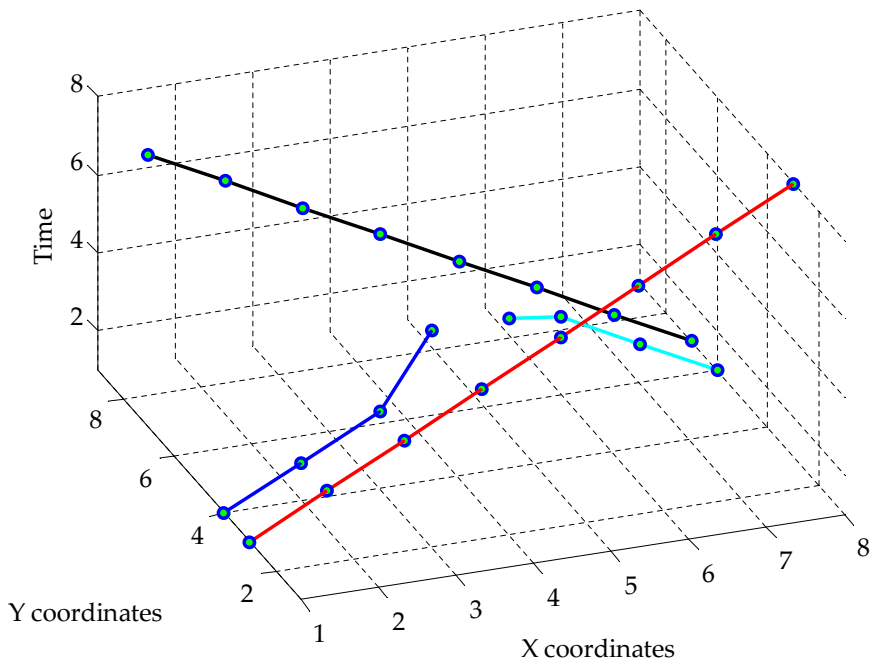

(b)

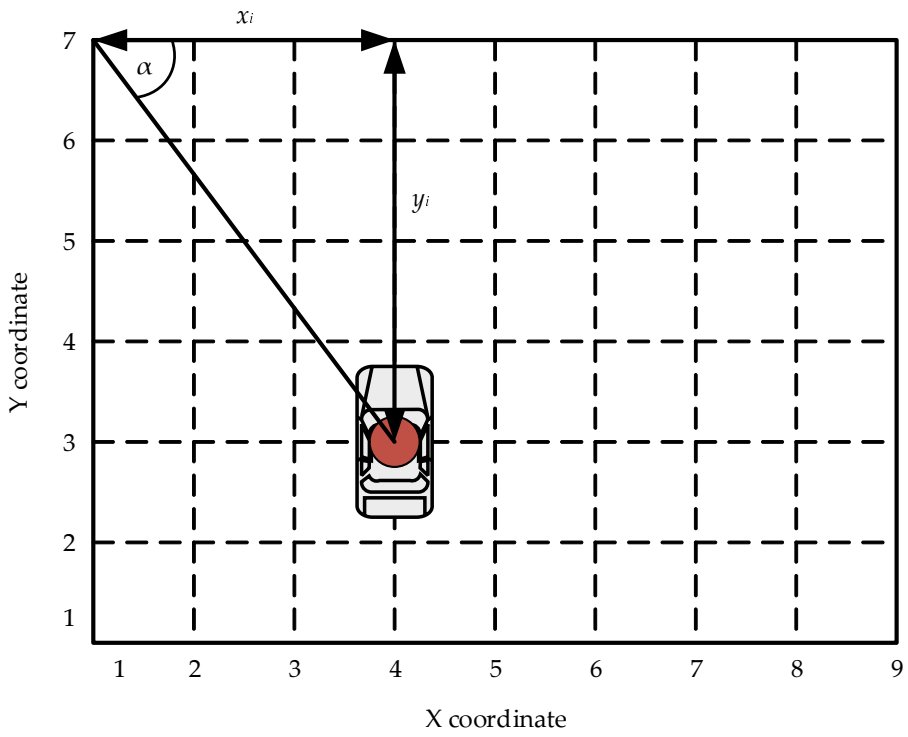

(c)

Figure 2. The object trajectory: (a) in the spatial dimension (b) in the temporal-spatial dimension; and (c) the direction of motion path. 
The features extracted from the video can be expressed as the feature-book $\mathcal{C}$ :

$$
\mathcal{C}=\left[\begin{array}{c}
\left(x_{1}^{1}, y_{1}^{1}, \alpha_{1}^{1}\right),\left(x_{1}^{2}, y_{1}^{2}, \alpha_{1}^{2}\right), \ldots,\left(x_{1}^{n}, y_{1}^{n}, \alpha_{1}^{n}\right) \\
\left(x_{2}^{1}, y_{2}^{1}, \alpha_{2}^{1}\right),\left(x_{2}^{2}, y_{2}^{2}, \alpha_{2}^{2}\right), \ldots,\left(x_{2}^{n}, y_{2}^{n}, \alpha_{2}^{n}\right) \\
\vdots \\
\left(x_{K}^{1}, y_{K}^{1}, \alpha_{K}^{1}\right),\left(x_{K}^{2}, y_{K}^{2}, \alpha_{K}^{2}\right), \ldots,\left(x_{K}^{n}, y_{K}^{n}, \alpha_{K}^{n}\right)
\end{array}\right]=\left[\begin{array}{c}
O_{1}^{n} \\
O_{2}^{n} \\
\vdots \\
O_{K}^{n}
\end{array}\right]
$$

where $K$ is the number of detected objects.

\subsection{Topic Modeling}

In this work, the traffic behavior is defined as the collection of activities in which an object trajectory is automatically assigned into an activity class. A short video can contain several trajectories that can be classified into the same activity class; i.e., they correlate in terms of location and/or direction. Therefore, it is important to model object trajectories in the correlative activities and to automatically model activities in the satisfactory behavior.

The pachinko allocation model (PAM) [35] is a hierarchical generative model considered here to define behaviors from the combination of features from moving objects. PAM was firstly suggested for use in the machine learning and natural language processing as a topic model. In its original application, PAM models correlations between topics in addition to word correlations and, thereby, establishes topics. To represent and learn arbitrary, nested and possibly sparse topic correlations, this model utilizes an arbitrary directed acyclic graph (DAGs) structure. Furthermore, compared to LDA [36], PAM provides more flexibility and greater expressive power than LDA, since it captures not only the correlations among the words, like in LDA, but also the correlations among topics.

In the following subsection, the details of the proposed model based on PAM are introduced with the algorithm for the estimation of the parameters. Although PAM employs arbitrary DAGs to model the topic correlations, this work proposes a four-level hierarchy structure as a special case of PAM [37]. This structure consists of one root topic, $u$ super topics at the second level $\mathcal{P}=\left\{p_{1}, p_{2}, \ldots, p_{u}\right\}, v$ subtopics at the third level $\mathcal{Q}=\left\{q_{1}, q_{2}, \ldots, q_{v}\right\}$ and the words at the bottom. Words refer here to the object features comprising the location and direction information, which were organized in the previous stage. The super topic and subtopic correspond to the traffic behavior and activity, respectively. The root is associated with behaviors; the behaviors are fully associated with activities; and the activities are fully connected to the features, as shown in Figure 3a. The multinomials of the root and behaviors are sampled for each frame based on a single Dirichlet distribution $g_{r}\left(\delta_{r}\right)$ and $\left.g_{j}\left(\delta_{j}\right)\right|_{j=1} ^{u}$, respectively. The activities are modeled with multinomial distributions $\left.\phi_{q_{j}}\right|_{j=1} ^{v}$ and $\left.\psi_{q_{j}}\right|_{j=1} ^{v}$ sampled from Dirichlet distribution $g(\beta)$ and $g(\gamma)$, which are used for sampling the location and direction features. Figure $3 \mathrm{~b}$ depicts a graphic model for the four-levels PAM. The particular notations used in PAM are summarized in Table 1. According to the standard PAM [35], considered a scene as a document $d$ consisting of a the sequence of $n$ frames $\mathcal{D}=\left\{d_{1}, d_{2}, \ldots, d_{n}\right\}$, this is modeled as follows:

1. Sample a multinomial distribution $\theta_{r}^{(d)}$ from a Dirichlet prior $\delta_{r}^{(d)}$ for each scene $d$.

2. For each behavior $p_{j}$, sample a multinomial distribution $\theta_{p_{j}}^{(d)}$ from $g_{j}\left(\delta_{j}\right)$ in which $\theta_{p_{j}}^{(d)}$ is a multinomial distribution over activities. 
3. Sample multinomial distributions $\left.\phi_{q_{j}}\right|_{j=1} ^{v}$ from a Dirichlet prior $\beta$ for each activity $q_{j}$.

4. Sample multinomial distributions $\left.\psi_{q_{j}}\right|_{j=1} ^{v}$ from a Dirichlet prior $\gamma$ for each activity $q_{j}$.

5. For the $m$-th feature in the current scene $d$ of the object $O_{k}$ :

- Sample a behavior $p_{m, d, O_{k}}$ from $\theta_{r}^{(d)}$ and an activity $q_{m, d, O_{k}}$ from $\theta_{p_{m, d, O_{k}}}^{(d)}$

- Sample a location feature $\chi_{m, d, O_{k}}$ from multinomial $\phi_{q_{m, d, O_{k}}}$ and a direction feature $\tau_{m, d, O_{k}}$ from multinomial $\psi_{q_{m, d, O_{k}}}$.

Following this process, the joint probability of the generated scene $d$, the behavior assignments $p^{(d)}$, the activity assignments $q^{(d)}$ and the multinomial distribution $\theta^{(d)}$ is calculated as:

$P\left(d, q^{(d)}, p^{(d)}, \theta^{(d)} \mid \delta, \beta, \gamma\right)=P\left(\theta_{r} \mid \delta_{r}\right) \prod_{j=1}^{u} P\left(\theta_{p_{j}}^{(d)} \mid \delta_{j}\right) \prod_{m}\left(P\left(p_{m} \mid \theta_{r}^{(d)}\right) P\left(q_{m} \mid \theta_{p_{m}}^{(d)}\right) P\left(f_{m} \mid \phi_{q}, \psi_{q}\right)\right)$

where $P\left(f_{m} \mid \phi_{q}, \psi_{q}\right)=P\left(\chi_{m} \mid \phi_{q}\right) P\left(\tau_{m} \mid \psi_{q}\right)$. Integrating out $\theta^{(d)}$ and summing over $p^{(d)}$ and $q^{(d)}$, the marginal probability of each scene can be calculated as:

$P(d \mid \delta, \beta, \gamma)=\int P\left(\theta_{r}^{(d)} \mid \delta_{r}\right) \prod_{j=1}^{u} P\left(\theta_{p_{j}}^{(d)} \mid \delta_{j}\right) \prod_{m} \sum_{p_{m}, q_{m}}\left(P\left(p_{m} \mid \theta_{r}^{(d)}\right) P\left(q_{m} \mid \theta_{p_{m}}^{(d)}\right) P\left(f_{m} \mid \phi_{q}, \psi_{q}\right)\right) d \theta^{(d)}$

The probability of generating the corpus $\mathcal{D}$ is computed by:

$$
P(\mathcal{D} \mid \delta, \beta, \gamma)=\int \prod_{j=1}^{v}\left(P\left(\phi_{q_{j}} \mid \beta\right)+P\left(\psi_{q_{j}} \mid \gamma\right)\right) \prod_{d} P(d \mid \delta, \beta, \gamma) d \phi d \psi
$$

The approximate inference result of the condition distribution that samples the behavior and activity assignments for each feature can be obtained as:

$$
\begin{aligned}
P\left(p_{m}, q_{m} \mid \mathcal{D}, \mathcal{P}_{-m}, \mathcal{Q}_{-m}, \delta, \beta, \gamma\right) & \propto P\left(m, p_{m}, q_{m} \mid \mathcal{D}_{-m}, \mathcal{P}_{-m}, \mathcal{Q}_{-m}, \delta, \beta, \gamma\right) \\
& =\frac{P(\mathcal{D}, \mathcal{P}, \mathcal{Q} \mid \delta, \beta, \gamma)}{P\left(\mathcal{D}, \mathcal{P}_{-m}, \mathcal{Q}_{-m} \mid \delta, \beta, \gamma\right)} \\
& =\frac{n_{j}^{(d)}+\delta_{r j}}{n_{r}^{(d)}+\sum_{j=1}^{u} \delta_{r j} n_{j}^{(d)}} \times \frac{n_{j l}^{(d)}+\delta_{j l}}{n_{j}^{(d)}+\sum_{l=1}^{v} \delta_{j l}} \times \frac{n_{l h}+\beta_{h}}{n_{l}+\sum_{h=1}^{N} \beta_{h}} \times \frac{n_{l z}+\gamma_{z}}{n_{l}+\sum_{z=1}^{M} \gamma_{z}}
\end{aligned}
$$

Hyper-parameters $\delta, \beta$ and $\gamma$ can be estimated via the Gibbs sampling algorithm, which is described in [35]. As in [35], the notation $-m$ denotes behavior assignments, except for the $m$-th feature. After modeling, the new data obtained by tagging the motion location and direction are generated. By merging the same feature items for different video contents, the probability distribution is obtained as an implicit activity-behavior-frame sequence matrix. The posterior is maximized by multiplying the direction probability of all locations from their corresponding subtopic location distributions. 


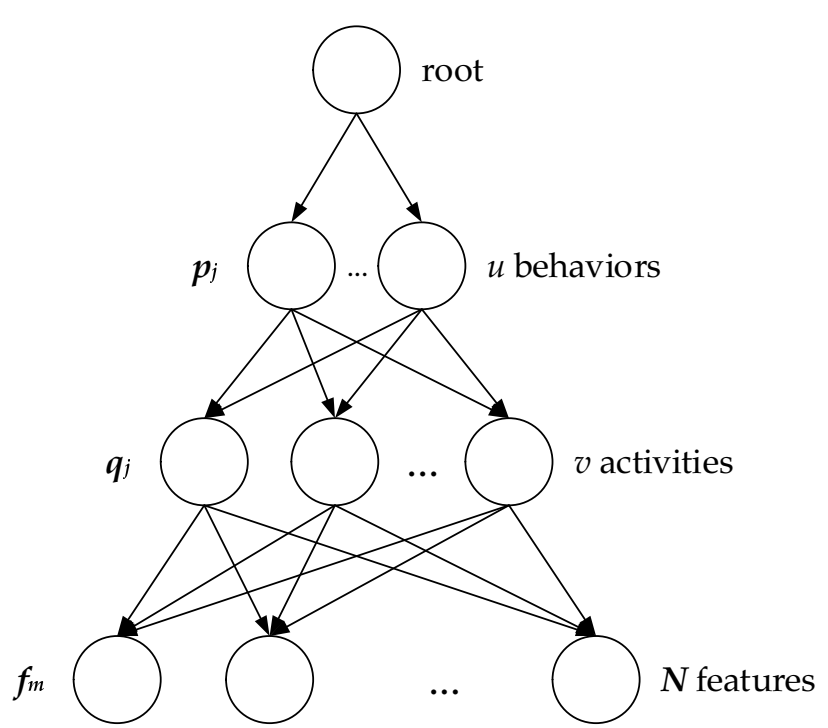

(a)

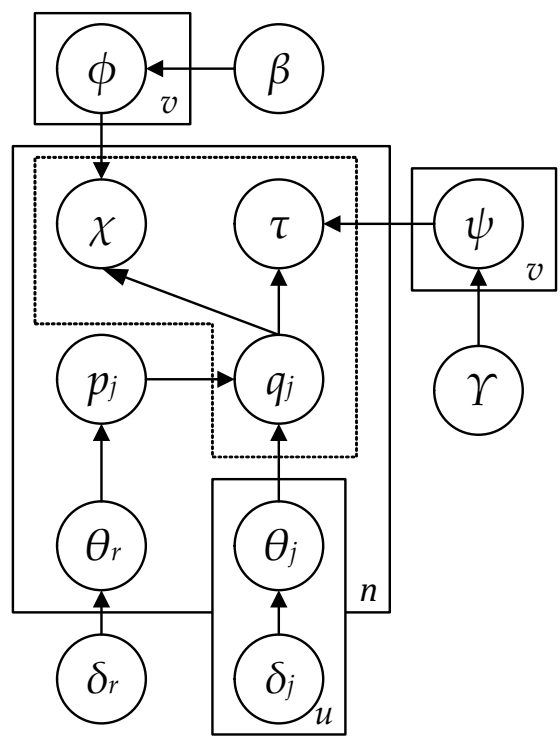

(b)

Figure 3. Pachinko allocation model: (a) hierarchical topic model (b) graphic model.

Table 1. Notations used in the pachinko allocation model (PAM) model.

\begin{tabular}{cc}
\hline Symbol & Description \\
\hline$u$ & Number of behaviors \\
$v$ & Number of activities \\
$n$ & Number of frames \\
$N$ & Number of unique locations \\
$M$ & Number of unique directions \\
$g_{r}\left(\delta_{r}\right)$ & Dirichlet distribution associated with the root \\
$g_{j}\left(\delta_{j}\right)$ & Dirichlet distribution associated with the $j$-th behavior, $u$-dimensional vector $g_{j}$ \\
$g(\beta)$ & Dirichlet distribution associated with activity for location feature \\
$g(\gamma)$ & Dirichlet distribution associated with activity for direction feature \\
$\theta_{r}^{(d)}$ & Multinomial distribution sampled from $g_{r}\left(\delta_{r}\right)$ for the root to scene $d, n$-dimensional vector $\theta_{r}$ \\
$\theta_{p_{j}}^{(d)}$ & Multinomial distribution sampled from $g_{j}\left(\delta_{j}\right)$ for a behavior to scene $d, u \times n$ matrix $\theta_{p}$ \\
$\phi_{q}$ & Multinomial distribution sampled from $g(\beta)$ for an activity to scene $d, v \times n$ matrix $\phi$ \\
$\psi_{q}$ & Multinomial distribution sampled from $g(\gamma)$ for an activity to scene $d, v \times n$ matrix $\psi$ \\
$\chi_{m, d, O_{k}}$ & $m$-th location in the frame $d$ of an object $O_{k}, N \times n \times k$ matrix $\chi$ \\
$\tau_{m, d, O_{k}}$ & $m$-th direction in the frame $d$ of an object $O_{k}, M \times n \times k$ matrix $\tau$ \\
$p_{m, d, O_{k}}$ & Behavior $p$ associated with the $m$-th feature in $d$ of $O_{k}, u$-dimensional vector $\mathcal{P}$ \\
$q_{m, d, O_{k}}$ & Activity $q$ associated with the $m$-th feature in $d$ of $O_{k}, v$-dimensional vector $\mathcal{Q}$ \\
$n_{r}^{(d)}$ & Number of occurrences of the root $r$ in the scene $d$ \\
$n_{j}^{(d)}$ & Notal number of occurrences of activity $q_{l}$ in the whole corpus $\mathcal{D}$ \\
$n_{l}$ & Number of occurrences of location feature $\chi_{h}$ in the activity $q_{l}$ \\
$n_{j l}^{(d)}$ & Number of occurrences of direction feature $\tau_{z}$ in the activity $q_{l}$ \\
$n_{l h}$ & $n_{l z}$
\end{tabular}




\subsection{Classification}

Based on the PAM-based topic modeling, every video sequence can be represented through a $u \times v$ matrix, where $u$ is the number of behaviors and $v$ is the number of activities. To train the classifier, the labels of vectors and matrices are manually denoted with their classes manually. In this paper, the authors use a SVM with binary tree architecture (SVM-BTA) [38] to solve the $N$-class pattern recognition problem. An illustration of SVM-BTA is shown in Figure 4. Each node in the architecture makes a binary decision using the original SVM. By recursively dividing the classes into two disjointed groups in each node of the decision tree, the SVM classifier decides the group to which the unknown samples that should be assigned. The class is determined by a clustering algorithm according to the class membership and the inter-class distance. Although $N-1 \mathrm{SVMs}$ are trained for an $N$-class problem, only $\log _{2} N$ SVMs are consulted at most to classify a sample. This approach requires fewer binary SVMs than popular methods, such as $N(N-1) / 2 \mathrm{SVMs}$ in the one-against-one approach and $N$ SVMs in the one-against-others approach. Moreover, both approaches have the drawback of very expensive computational cost requirements and accuracy degradation [38]. An essential contribution of the SVM-BTA approach, the multiclass issue, is converted into binary-tree architectures without performance reduction. Moreover, a dramatic improvement in recognition speed can be achieved for increasing the number of classes.

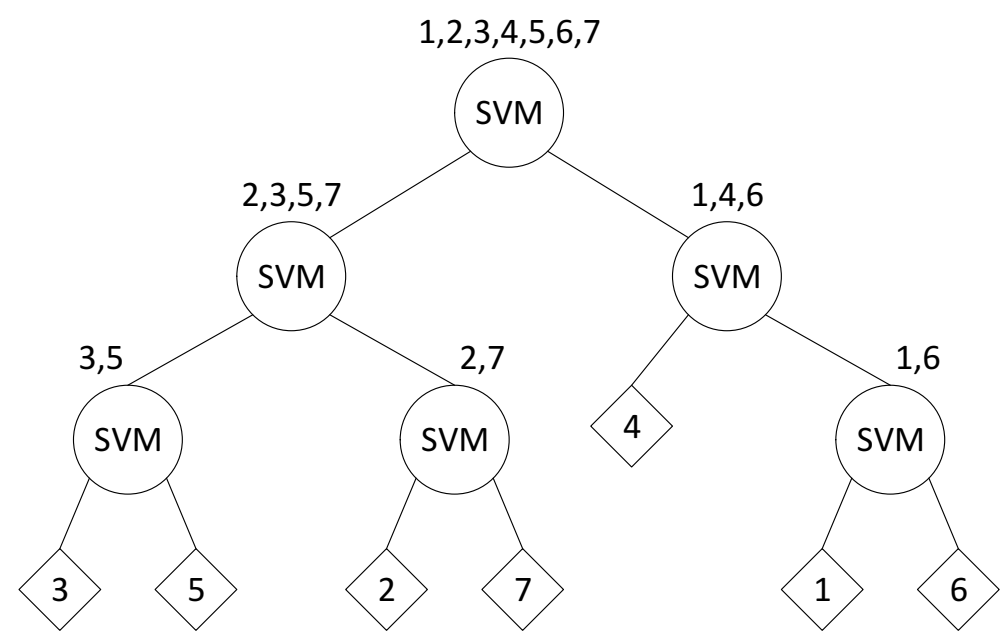

Figure 4. Illustration of SVM-binary tree architecture (BTA).

\section{Experimental Evaluation}

\subsection{Experimental Setup}

The experiments were performed on the QMUL (Queen Mary University of London) dataset [39], which includes a long-term video recorded at $25 \mathrm{fps}$ for the frame rate and $360 \times 288$ for the frame resolution. Placed at an intersection, the video captured a busy traffic scenario involving a vehicle and pedestrian with dynamic movements. The video sequence was divided into short non-overlapping clips, each of which was $4 \mathrm{~s}$. This duration is more convenient for observing when compared with too long 
a duration in Hospedales's work [40] (12 s) or two short a duration in Zhao's work [16] (2 s). The length of each clip was set to ensure that a behavior was not covered by others. A total of 750 clips comprised 320 vertical traffic flow clips; 430 horizontal traffic flow clips were tested with the manual activity and behavior labeling. Some activities cannot be fully categorized into horizontal or vertical traffic behavior, for example 40 frames may represent vertical traffic and 60 frames horizontal traffic. For example, a car can move in the vertical traffic from the top, and it will turn left or turn right at the intersection. Therefore, the authors categorized a given clip into either vertical or horizontal behavior based on the duration of the observed behaviors. If both behaviors are present during the whole clip, this is categorized into the most fluent behavior, i.e., with less changes or interruptions. In the vertical traffic, activities were discovered by PAM, as shown in Figure 5a-c. The horizontal traffic activities are presented in Figure 5d-h. Although PAM automatically discovered and modeled sparse words into super topics and subtopics, the number of topics had to be initially set. In this work, $u=2$ for vertical and horizontal traffic behaviors; and $v=14$ for traffic activities involving six vertical and eight horizontal activities. The description of the discovered activities outlined in Figure 5 is referenced in Table 2. In the PAM modeling, the Dirichlet distribution over behaviors and activities was produced with the parameter 0.01; the Gibbs sampling was processed with 1000 burn-in iterations. In the SVM-BTA classifier, the Gaussian kernel was used to set up for each node of binary classification. For each vertical and horizontal traffic dataset, the proposed method was evaluated using the 10-fold cross-validation. In order to analyze accuracy of the proposed method, Recall and Precision are used with the confusion matrix of each experiment. All of the experiments were performed on a desktop PC operating Windows 7 with a 2.67-GHz Intel Core i5 CPU and 4 GB of RAM. MATLAB R2013a was the software used for simulation.

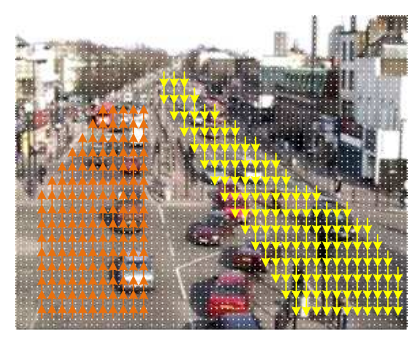

(a)

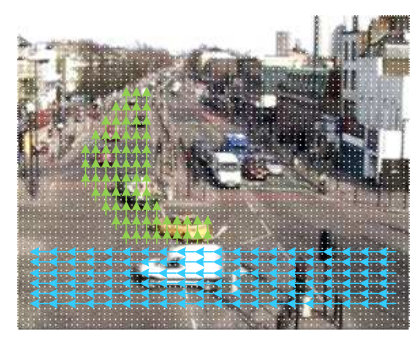

(e)

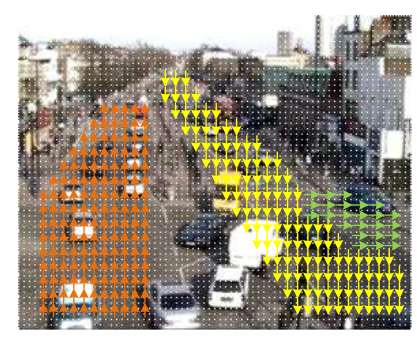

(b)

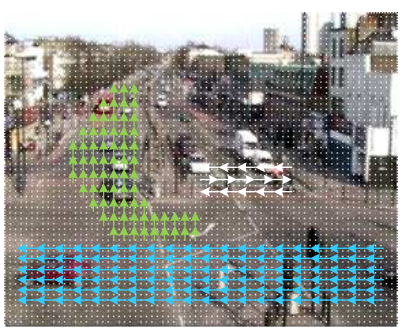

(f)

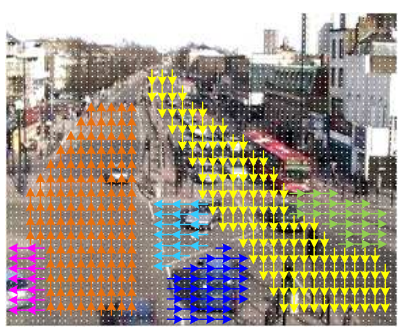

(c)

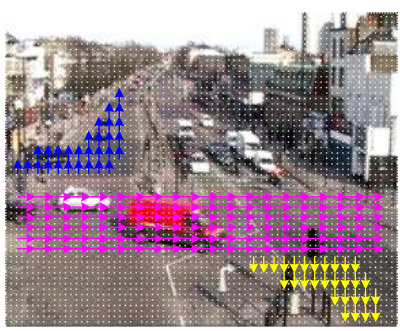

$(\mathrm{g})$

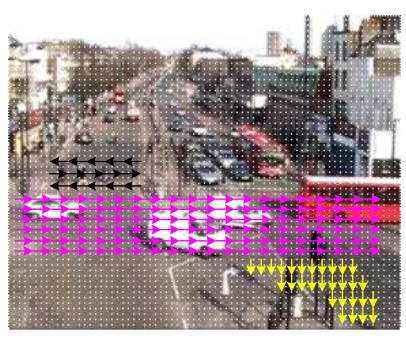

(d)

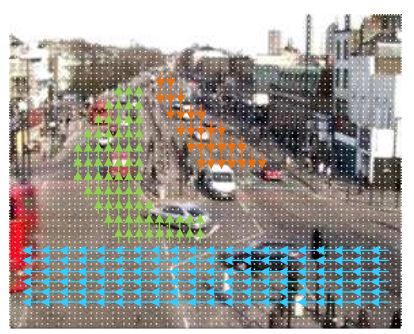

(h)

Figure 5. Traffic activities discovered by PAM. $(\mathbf{a}-\mathbf{c})$ The vertical traffic behavior; $(\mathbf{d}-\mathbf{h})$ the horizontal traffic behavior. 
Table 2. Activity descriptions of two main behaviors.

\begin{tabular}{cccc}
\hline & \multicolumn{3}{c}{ Vertical Traffic } \\
\hline Activity & Color & Figure 5 & Description \\
\hline V1 & Orange & (a), (b), (c) & Bottom to top flow \\
V2 & Blue & (c) & Bottom to top and turn left at the intersection \\
V3 & Pink & (c) & Bottom to top and turn right at the intersection \\
V4 & Yellow & (a), (b), (c) & \multicolumn{1}{c}{ Top to bottom flow } \\
V5 & Green & (b), (c) & Top to bottom and turn left at the intersection \\
V6 & Cyan & (c) & Top to bottom and turn right at the intersection \\
\hline & & & Horizontal Traffic \\
\hline Activity & Color & Figure 5 & Description \\
\hline H1 & Black & (d) & Vertical flow for pedestrian on the left side \\
H2 & White & (f) & Vertical flow for pedestrian on the right side \\
H3 & Pink & (d), (g) & Left to right flow \\
H4 & Yellow & (d), (g) & Left to right and turn right at the intersection \\
H5 & Blue & (g) & Left to right and turn left at the intersection \\
H6 & Cyan & (e), (f), (h) & Right to left flow \\
H7 & Green & (e), (f), (h) & Right to left and turn right at the intersection \\
H8 & Orange & (h) & Top to bottom and stop at the intersection \\
\hline
\end{tabular}

\subsection{Results and Discussion}

In the experiments, the authors evaluated the performance in the classification accuracy of the proposed method for the detection of the vertical and horizontal traffic. Moreover, the method was compared with similar approaches using standard latent Dirichlet allocation (LDA) [36] and Markov clustering topic mode (MCTM) [30] for topic modeling. At first, the activity classification was applied to each separate dataset of the vertical and horizontal clips. The confusion matrices of the SVM-BTA classifier using PAM and LDA are reported in Tables 3-5 for the vertical and in Tables 6-8 for the horizontal traffic dataset. The mixture of all vertical and horizontal traffic activity classification results are presented in the confusion matrix shown in Figure 6 with 14 classes in total. Secondly, the behavior classification was evaluated for all clips to identify the category of the input clip. For behavior classification, all clips in the merged dataset were evaluated using the binary SVM classifier. The quantitative results of the evaluated metrics are represented in Table 9. It is important to note that only the binary SVM classifier was utilized for the behavior classification (either vertical or horizontal) instead of the multi-class SVM classifier for the activity case. 
Table 3. Confusion matrix of the SVM classifier using PAM for the vertical traffic.

\begin{tabular}{cccccccc}
\hline Activities & V1 & V2 & V3 & V4 & V5 & V6 & Recall (\%) \\
\hline V1 & 93 & 5 & 3 & 0 & 0 & 0 & 91.18 \\
V2 & 2 & 26 & 0 & 0 & 0 & 0 & 92.86 \\
V3 & 1 & 0 & 19 & 0 & 0 & 0 & 95.00 \\
V4 & 0 & 2 & 0 & 97 & 6 & 5 & 88.18 \\
V5 & 0 & 0 & 0 & 4 & 38 & 0 & 90.48 \\
V6 & 0 & 0 & 0 & 1 & 0 & 17 & 94.44 \\
\hline Precision (\%) & 96.88 & 78.79 & 86.36 & 95.10 & 86.36 & 73.91 & \\
\hline Accuracy (\%) & & & \multicolumn{7}{c}{$\mathbf{9 0 . 6 3}$} \\
\hline
\end{tabular}

Table 4. Confusion matrix of the SVM classifier using LDA for the vertical traffic.

\begin{tabular}{cccccccc}
\hline Activities & V1 & V2 & V3 & V4 & V5 & V6 & Recall (\%) \\
\hline V1 & 89 & 4 & 1 & 7 & 0 & 1 & 87.25 \\
V2 & 0 & 25 & 0 & 3 & 0 & 0 & 89.29 \\
V3 & 3 & 0 & 17 & 0 & 0 & 0 & 85.00 \\
V4 & 9 & 2 & 0 & 88 & 8 & 3 & 80.00 \\
V5 & 0 & 0 & 3 & 4 & 35 & 0 & 83.33 \\
V6 & 0 & 0 & 0 & 3 & 0 & 15 & 83.33 \\
\hline Precision (\%) & 88.12 & 80.65 & 80.95 & 83.81 & 81.40 & 78.95 & \\
\hline Accuracy (\%) & & \multicolumn{7}{c}{$\mathbf{8 4 . 0 6}$} \\
\hline
\end{tabular}

Table 5. Confusion matrix of the SVM classifier using Markov clustering topic model (MCTM) for the vertical traffic.

\begin{tabular}{cccccccc}
\hline Activities & V1 & V2 & V3 & V4 & V5 & V6 & Recall (\%) \\
\hline V1 & 92 & 4 & 2 & 3 & 0 & 1 & 90.20 \\
V2 & 1 & 27 & 0 & 0 & 0 & 0 & 96.43 \\
V3 & 5 & 0 & 15 & 0 & 0 & 0 & 75.00 \\
V4 & 5 & 0 & 0 & 105 & 2 & 2 & 95.45 \\
V5 & 0 & 0 & 3 & 3 & 36 & 0 & 85.71 \\
V6 & 0 & 0 & 1 & 1 & 0 & 16 & 88.89 \\
\hline Precision (\%) & 92.93 & 87.10 & 71.43 & 93.75 & 94.74 & 84.21 & \\
\hline Accuracy (\%) & & & $\mathbf{9 0 . 9 4}$ \\
\hline
\end{tabular}


Table 6. Confusion matrix of the SVM classifier using PAM for the horizontal traffic.

\begin{tabular}{cccccccccc}
\hline Activities & H1 & H2 & H3 & H4 & H5 & H6 & H7 & H8 & Recall (\%) \\
\hline H1 & 11 & 0 & 0 & 0 & 1 & 0 & 0 & 0 & 91.67 \\
H2 & 0 & 9 & 0 & 0 & 0 & 0 & 0 & 1 & 90.00 \\
H3 & 0 & 0 & 112 & 2 & 0 & 9 & 3 & 0 & 88.89 \\
H4 & 0 & 0 & 0 & 31 & 0 & 3 & 0 & 0 & 91.18 \\
H5 & 1 & 0 & 0 & 0 & 18 & 0 & 1 & 0 & 90.00 \\
H6 & 0 & 0 & 12 & 0 & 0 & 125 & 5 & 0 & 88.03 \\
H7 & 0 & 0 & 0 & 0 & 4 & 0 & 60 & 0 & 93.75 \\
H8 & 0 & 1 & 0 & 0 & 0 & 0 & 0 & 21 & 95.45 \\
\hline Precision (\%) & 91.67 & 90.00 & 90.32 & 93.94 & 78.26 & 91.24 & 86.96 & 95.45 & \\
\hline Accuracy (\%) & & & & \multicolumn{7}{c}{$\mathbf{9 0 . 0 0}$} \\
\hline
\end{tabular}

Table 7. Confusion matrix of the SVM classifier using LDA for the horizontal traffic.

\begin{tabular}{cccccccccc}
\hline Activities & H1 & H2 & H3 & H4 & H5 & H6 & H7 & H8 & Recall (\%) \\
\hline H1 & 11 & 0 & 0 & 0 & 1 & 0 & 0 & 0 & 91.67 \\
H2 & 0 & 9 & 0 & 0 & 0 & 0 & 0 & 1 & 90.00 \\
H3 & 1 & 1 & 106 & 4 & 0 & 11 & 3 & 0 & 84.13 \\
H4 & 0 & 0 & 0 & 28 & 0 & 6 & 0 & 0 & 82.35 \\
H5 & 1 & 0 & 1 & 0 & 17 & 0 & 1 & 0 & 85.00 \\
H6 & 0 & 0 & 10 & 5 & 0 & 119 & 8 & 0 & 83.80 \\
H7 & 0 & 0 & 0 & 0 & 7 & 0 & 57 & 0 & 89.06 \\
H8 & 0 & 1 & 0 & 0 & 0 & 0 & 0 & 21 & 95.45 \\
\hline Precision $(\%)$ & 84.62 & 81.82 & 90.60 & 75.68 & 68.00 & 87.50 & 82.61 & 95.45 & \\
\hline Accuracy $(\%)$ & & & & \multicolumn{7}{c}{$\mathbf{8 5 . 5 8}$} \\
\hline
\end{tabular}

Table 8. Confusion matrix of the SVM classifier using MCTM for the horizontal traffic.

\begin{tabular}{cccccccccc}
\hline Activities & H1 & H2 & H3 & H4 & H5 & H6 & H7 & H8 & Recall (\%) \\
\hline H1 & 10 & 0 & 1 & 0 & 1 & 0 & 0 & 0 & 83.33 \\
H2 & 0 & 9 & 0 & 0 & 0 & 0 & 0 & 1 & 90.00 \\
H3 & 1 & 1 & 111 & 4 & 0 & 6 & 3 & 0 & 88.10 \\
H4 & 0 & 0 & 2 & 26 & 0 & 6 & 0 & 0 & 76.47 \\
H5 & 1 & 0 & 1 & 0 & 17 & 0 & 1 & 0 & 85.00 \\
H6 & 0 & 0 & 5 & 3 & 3 & 128 & 3 & 0 & 90.14 \\
H7 & 0 & 0 & 0 & 0 & 7 & 0 & 57 & 0 & 89.06 \\
H8 & 0 & 1 & 0 & 0 & 0 & 0 & 0 & 21 & 95.45 \\
\hline Precision $(\%)$ & 83.33 & 81.82 & 92.50 & 78.79 & 60.70 & 91.43 & 89.06 & 95.45 & \\
\hline Accuracy $(\%)$ & & & & & $\mathbf{8 8 . 1 4}$ & & & \\
\hline
\end{tabular}


Table 9. Behavior classification comparison between PAM and LDA.

\begin{tabular}{|c|c|c|c|c|c|c|c|c|c|}
\hline \multirow[b]{2}{*}{ Behavior } & \multicolumn{3}{|c|}{ PAM } & \multicolumn{3}{|c|}{ LDA [36] } & \multicolumn{3}{|c|}{ MCTM [30] } \\
\hline & Vertical & Horizontal & Recall (\%) & Vertical & Horizontal & Recall (\%) & Vertical & Horizontal & Recall (\%) \\
\hline Vertical & 286 & 34 & 89.38 & 259 & 61 & 80.94 & 291 & 29 & 90.94 \\
\hline Horizontal & 59 & 371 & 86.28 & 66 & 364 & 84.65 & 47 & 383 & 89.07 \\
\hline Precision (\%) & 82.90 & 91.60 & - & 79.69 & 85.65 & - & 86.09 & 92.96 & - \\
\hline Accuracy (\%) & & 87.60 & & & 83.07 & & & 89.87 & \\
\hline
\end{tabular}

$\begin{array}{lllllllllllllllll}\text { V1 } & 0.84 & 0.04 & 0.03 & 0.00 & 0.00 & 0.00 & 0.00 & 0.00 & 0.00 & 0.00 & 0.03 & 0.00 & 0.06 & 0.00\end{array}$

$\begin{array}{lllllllllllllll}\text { V2 } & 0.07 & 0.86 & 0.00 & 0.00 & 0.00 & 0.00 & 0.00 & 0.00 & 0.04 & 0.00 & 0.00 & 0.04 & 0.00 & 0.00\end{array}$

$\begin{array}{lllllllllllllll}\text { V3 } & 0.05 & 0.00 & 0.85 & 0.00 & 0.00 & 0.00 & 0.00 & 0.00 & 0.00 & 0.00 & 0.00 & 0.10 & 0.00 & 0.00\end{array}$

$\begin{array}{lllllllllllllll}\text { V4 } & 0.00 & 0.02 & 0.00 & 0.85 & 0.04 & 0.05 & 0.00 & 0.00 & 0.00 & 0.00 & 0.00 & 0.00 & 0.00 & 0.05\end{array}$

$\begin{array}{lllllllllllllll}\text { V5 } & 0.00 & 0.00 & 0.00 & 0.05 & 0.90 & 0.00 & 0.00 & 0.00 & 0.05 & 0.00 & 0.00 & 0.00 & 0.00 & 0.00\end{array}$

$\begin{array}{lllllllllllllll}\text { V6 } & 0.00 & 0.00 & 0.00 & 0.06 & 0.00 & 0.89 & 0.00 & 0.00 & 0.00 & 0.00 & 0.00 & 0.00 & 0.00 & 0.06\end{array}$

$\begin{array}{lllllllllllllll}\mathrm{H} 1 & 0.00 & 0.00 & 0.00 & 0.00 & 0.00 & 0.00 & 0.92 & 0.00 & 0.00 & 0.00 & 0.08 & 0.00 & 0.00 & 0.00\end{array}$

$\begin{array}{lllllllllllllll}\mathrm{H} 2 & 0.00 & 0.00 & 0.00 & 0.00 & 0.00 & 0.00 & 0.00 & 0.90 & 0.00 & 0.00 & 0.00 & 0.00 & 0.00 & 0.10\end{array}$

$\begin{array}{lllllllllllllll}\text { H3 } & 0.00 & 0.00 & 0.00 & 0.00 & 0.02 & 0.02 & 0.00 & 0.00 & 0.87 & 0.02 & 0.00 & 0.05 & 0.02 & 0.00\end{array}$

$\begin{array}{lllllllllllllll}\mathrm{H} 4 & 0.00 & 0.00 & 0.00 & 0.06 & 0.00 & 0.00 & 0.00 & 0.00 & 0.00 & 0.88 & 0.00 & 0.06 & 0.00 & 0.00\end{array}$

H5 $\begin{array}{lllllllllllllll}0.00 & 0.00 & 0.00 & 0.00 & 0.00 & 0.00 & 0.05 & 0.00 & 0.00 & 0.00 & 0.80 & 0.00 & 0.05 & 0.00\end{array}$

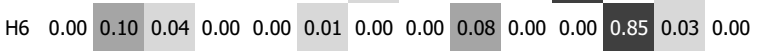

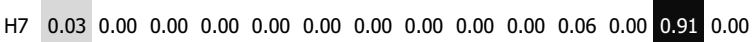

$\begin{array}{lllllllllllllll}\text { H8 } & 0.00 & 0.09 & 0.00 & 0.00 & 0.00 & 0.00 & 0.00 & 0.05 & 0.00 & 0.00 & 0.00 & 0.00 & 0.00 & 0.86\end{array}$

$\begin{array}{llllllllllllll}\mathrm{V} 1 & \mathrm{~V} 2 & \mathrm{~V} 3 & \mathrm{~V} 4 & \mathrm{~V} 5 & \mathrm{~V} 6 & \mathrm{H} 1 & \mathrm{H} 2 & \mathrm{H} 3 & \mathrm{H} 4 & \mathrm{H} 5 & \mathrm{H} 6 & \mathrm{H} 7 & \mathrm{H} 8\end{array}$

(a) $\begin{array}{llllllllllllllll}\text { V1 } & 0.81 & 0.04 & 0.03 & 0.04 & 0.00 & 0.00 & 0.00 & 0.00 & 0.00 & 0.00 & 0.06 & 0.00 & 0.02 & 0.00\end{array}$

$\begin{array}{lllllllllllllll}\text { V2 } & 0.04 & 0.82 & 0.00 & 0.04 & 0.00 & 0.00 & 0.00 & 0.00 & 0.07 & 0.00 & 0.00 & 0.04 & 0.00 & 0.00\end{array}$

$\begin{array}{lllllllllllllll}\text { V3 } & 0.10 & 0.00 & 0.80 & 0.00 & 0.00 & 0.00 & 0.00 & 0.00 & 0.00 & 0.00 & 0.00 & 0.10 & 0.00 & 0.00\end{array}$ $\begin{array}{lllllllllllllll}\text { V4 } & 0.00 & 0.02 & 0.00 & 0.77 & 0.07 & 0.05 & 0.00 & 0.00 & 0.00 & 0.03 & 0.00 & 0.00 & 0.00 & 0.06\end{array}$ $\begin{array}{lllllllllllllll}\text { V5 } & 0.00 & 0.00 & 0.05 & 0.05 & 0.81 & 0.00 & 0.00 & 0.00 & 0.10 & 0.00 & 0.00 & 0.00 & 0.00 & 0.00\end{array}$ $\begin{array}{lllllllllllllll}\text { V6 } & 0.00 & 0.00 & 0.00 & 0.11 & 0.00 & 0.83 & 0.00 & 0.00 & 0.00 & 0.00 & 0.00 & 0.00 & 0.00 & 0.06\end{array}$ $\begin{array}{lllllllllllllll}\mathrm{H} 1 & 0.00 & 0.00 & 0.00 & 0.00 & 0.00 & 0.00 & 0.92 & 0.00 & 0.00 & 0.00 & 0.08 & 0.00 & 0.00 & 0.00\end{array}$ $\begin{array}{lllllllllllllll}\text { H2 } & 0.00 & 0.00 & 0.00 & 0.00 & 0.00 & 0.00 & 0.00 & 0.70 & 0.20 & 0.00 & 0.00 & 0.00 & 0.00 & 0.10\end{array}$ $\begin{array}{lllllllllllllll}\mathrm{H} 3 & 0.00 & 0.00 & 0.00 & 0.00 & 0.03 & 0.02 & 0.00 & 0.02 & 0.82 & 0.03 & 0.00 & 0.08 & 0.02 & 0.00\end{array}$ $\begin{array}{lllllllllllllll}\mathrm{H} 4 & 0.00 & 0.00 & 0.00 & 0.06 & 0.00 & 0.00 & 0.00 & 0.00 & 0.00 & 0.88 & 0.00 & 0.06 & 0.00 & 0.00\end{array}$ H5 $\begin{array}{lllllllllllllll}0.10 & 0.00 & 0.00 & 0.00 & 0.00 & 0.00 & 0.05 & 0.00 & 0.00 & 0.00 & 0.80 & 0.00 & 0.05 & 0.00\end{array}$ $\begin{array}{lllllllllllllllll}\text { H6 } & 0.00 & 0.00 & 0.06 & 0.00 & 0.00 & 0.03 & 0.00 & 0.00 & 0.08 & 0.04 & 0.00 & 0.73 & 0.06 & 0.00\end{array}$

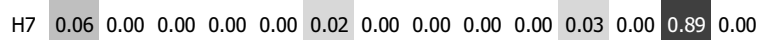
$\begin{array}{lllllllllllllll}\text { H8 } & 0.00 & 0.09 & 0.00 & 0.00 & 0.00 & 0.00 & 0.00 & 0.05 & 0.00 & 0.00 & 0.00 & 0.00 & 0.00 & 0.86\end{array}$ $\begin{array}{llllllllllllll}\text { V1 } & \text { V2 } & \text { V3 } & \text { V4 } & \text { V5 } & \text { V6 } & \text { H1 } & \text { H2 } & \text { H3 } & \text { H4 } & \text { H5 } & \text { H6 } & \text { H7 } & \text { H8 }\end{array}$

(b)

$\begin{array}{llllllllllllllll}\text { V1 } & 0.84 & 0.05 & 0.02 & 0.04 & 0.00 & 0.00 & 0.00 & 0.00 & 0.00 & 0.00 & 0.03 & 0.00 & 0.02 & 0.00\end{array}$

$\begin{array}{llllllllllllllll}\text { V2 } & 0.04 & 0.82 & 0.00 & 0.04 & 0.00 & 0.00 & 0.00 & 0.00 & 0.07 & 0.00 & 0.00 & 0.04 & 0.00 & 0.00\end{array}$

$\begin{array}{lllllllllllllll}\text { V3 } & 0.15 & 0.00 & 0.80 & 0.00 & 0.00 & 0.00 & 0.00 & 0.00 & 0.00 & 0.00 & 0.00 & 0.05 & 0.00 & 0.00\end{array}$

$\begin{array}{lllllllllllllll}\text { V4 } & 0.00 & 0.02 & 0.00 & 0.78 & 0.06 & 0.05 & 0.00 & 0.00 & 0.00 & 0.03 & 0.00 & 0.00 & 0.00 & 0.06\end{array}$

$\begin{array}{lllllllllllllll}\text { V5 } & 0.00 & 0.00 & 0.05 & 0.05 & 0.83 & 0.00 & 0.00 & 0.00 & 0.07 & 0.00 & 0.00 & 0.00 & 0.00 & 0.00\end{array}$

$\begin{array}{lllllllllllllll}\text { V6 } & 0.00 & 0.00 & 0.00 & 0.11 & 0.00 & 0.83 & 0.00 & 0.00 & 0.00 & 0.00 & 0.00 & 0.00 & 0.00 & 0.06\end{array}$

H1 $\begin{array}{llllllllllllll}0.00 & 0.00 & 0.00 & 0.00 & 0.00 & 0.00 & 0.92 & 0.00 & 0.00 & 0.00 & 0.08 & 0.00 & 0.00 & 0.00\end{array}$

H2 $\begin{array}{lllllllllllllll} & 0.00 & 0.00 & 0.00 & 0.00 & 0.00 & 0.00 & 0.00 & 0.60 & 0.20 & 0.00 & 0.00 & 0.00 & 0.00 & 0.20\end{array}$

H3 $\begin{array}{llllllllllllllll} & 0.00 & 0.00 & 0.00 & 0.00 & 0.04 & 0.00 & 0.00 & 0.02 & 0.83 & 0.02 & 0.00 & 0.06 & 0.02 & 0.00\end{array}$

$\begin{array}{lllllllllllllll}\mathrm{H} 4 & 0.00 & 0.00 & 0.00 & 0.12 & 0.00 & 0.00 & 0.00 & 0.00 & 0.00 & 0.82 & 0.00 & 0.06 & 0.00 & 0.00\end{array}$

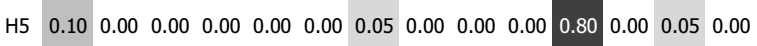

H6 $\begin{array}{llllllllllllllll}0.00 & 0.00 & 0.06 & 0.00 & 0.00 & 0.04 & 0.00 & 0.00 & 0.05 & 0.04 & 0.00 & 0.75 & 0.06 & 0.00\end{array}$

H7 $\begin{array}{lllllllllllllll}0.05 & 0.00 & 0.00 & 0.00 & 0.00 & 0.02 & 0.00 & 0.00 & 0.00 & 0.00 & 0.02 & 0.00 & 0.92 & 0.00\end{array}$

H8 $\begin{array}{lllllllllllllllll}0.00 & 0.09 & 0.00 & 0.00 & 0.00 & 0.00 & 0.00 & 0.05 & 0.00 & 0.00 & 0.00 & 0.00 & 0.00 & 0.86\end{array}$

$\begin{array}{llllllllllllll}\text { V1 } & \text { V2 } & \text { V3 } & \text { V4 } & \text { V5 } & \text { V6 } & \text { H1 } & \text { H2 } & \text { H3 } & \text { H4 } & \text { H5 } & \text { H6 } & \text { H7 } & \text { H8 }\end{array}$

(c)

Figure 6. Confusion matrix of the SVM classifier for the mixing of all vertical and horizontal traffic with overall classification accuracy: (a) PAM 86.4\%; (b) LDA $80.4 \%$; and (c) MCTM 81.6\%. 
In the vertical and horizontal traffic datasets, the numbers of clips presenting particular activities discovered by PAM were not equivalent. For example, the occurrence of activity V1 and V4 in the vertical dataset corresponding to the top-bottom flows consumed more than $66 \%$ of the full video length. Similarly, activities $\mathrm{H} 3$ and $\mathrm{H} 6$ in the horizontal dataset corresponding to left-right flows consumed more than approximately $62 \%$ of the video length. Therefore, they can be regarded as the primary activities corresponding to each dataset. Based on the results in the Table 3, activities V3 and V6 obtained the highest accuracies (greater than 94\%), because they contained specific features in the given direction. On the other hand, activities V4 and V5 had the worst classification rates, since they easily overlapped by covering similar location and direction features. Six clips were recognized as V5, and five clips were recognized as V6 instead of the correct class of V4. It was evident that the primary activities with high appearance frequencies had larger interesting regions compared with the others. Some small regions with fewer appearance activities were covered by them, which resulted in the classification confusion. This phenomenon likewise occurred in the horizontal dataset with the worst classification results of the primary activities, particularly activities $\mathrm{H} 3$ and $\mathrm{H} 6$ in Table 5.

In the activity classification using the respective vertical and horizontal traffic clips separately, the proposed method using PAM for topic modeling outperformed the LDA-based method in most tested activities (above $90 \%$ of overall accuracy rate). Compared with LDA, PAM provided the higher accuracy rate through the Recall and Precision metrics, especially with the overall Accuracy (greater than 6\% in the vertical dataset and $4 \%$ in the horizontal dataset). Although obtaining a high classification performance, MCTM overall accuracy degraded along with the increase of the number of classes ( $90.94 \%$ for six vertical classes compared with $88.14 \%$ for eight horizontal classes). When merging the vertical and horizontal dataset for activity classification, the accuracy tended to reduce in all evaluated models. From the results in Figure 6, PAM outperformed LDA and MCTM with $86.4 \%$ vs. 80.4\% and $81.6 \%$ in terms of Accuracy. When the number of classes was increased in the merging dataset, a strong degradation was again observed in the MCTM model, because it is difficult to correctly classify activities of two or more phases, for example activity V5 including one part of a common activity (top to bottom flow) and another part of unusual activity (turning left at the intersection). MCTM got only $60 \%$ in terms of accuracy with rare activity $\mathrm{H} 2$, while some common activities are confused with others, such as activity V4 with V5 and H8 and activity H6 with V3 and H7. In the merging of all clips to classify the behavior, MCTM still showed the highest accuracy rate. Concretely, only 76 clips $(\approx 10.1 \%)$ were incorrectly recognized by MCTM instead of 93 and 127 clips $(\approx 12.4 \%$ and $16.9 \%$ ) respectively misclassified by PAM and LDA. Despite using the DAGs structure and exploiting the Dirichlet distribution, LDA only captured the correlation among the features to support the high level information (activities or behaviors), because it was constructed by a three-layer model comprising the feature, activity (or behavior) and root layers. Therefore, LDA has difficulty modeling data in which some behaviors co-occurred more frequently than in others. Compared with PAM capturing only spatial correlation between activities, MCTM further measured temporal correlation between events to improve the binary behavior classification. Moreover, the distinction between only two vertical and horizontal behaviors is more explicit under the spatio-temporal dimension. However, MCTM sometimes had a negative effect for allowing a rare activity to occur alongside numerous common activities [30]. This led 
to the degradation of the classification accuracy in the MCTM model whenever the number of behaviors or activities was increased.

\subsection{Complexity and Computational Time}

It is difficult to provide theoretical analysis for the convergence of Gibbs sampling. Concretely, the time complexity of each Gibbs sampling iteration for LDA was $\mathcal{O}(N u)+\mathcal{O}(N v)$ when modeling $N$ features into $u$ behaviors and $v$ activities. Running on our system, it took less than $2 \mathrm{~h}$ to process 750 4-s clips from the UMUL dataset. The Gibbs sampling for PAM was much slower at approximately $3.5 \mathrm{~h}$, because it depended not only on the number of super topics, but also on the number of sub-topics with $\mathcal{O}(N u v)$. Since two layers, including action and behavior, were considered in the MCTM model, it required $\mathcal{O}\left(u^{2}\right)+\mathcal{O}(N u v)$ time per parameter sample [30]. The total process time of MCTM was quite equivalent to PAM's time owing to the insignificance of the component $\mathcal{O}\left(u^{2}\right)$ in comparison to $\mathcal{O}(N u v)$ of the proposed method.

\section{Conclusions}

In this paper, the authors proposed a behavior recognition method based on a four-level hierarchy PAM model for traffic video surveillance purposes. Two types of features extracted from a traffic video, comprising the location and direction of the moving object, are used to construct the object trajectory. For topic modeling, the PAM algorithm is then used to reorganize the sparse features. The probability distribution, the new data generated from PAM, is then provided for the SVM-BTA classifier. With the advantage of capturing correlations among features, as well as among activities and behaviors, PAM provides more expressive power to support complicated structures, while adopting more realistic assumptions. This property helps improve the classification rate in behavior recognition. In our experimental evaluation, the proposed method is compared with LDA and MCTM in individual datasets of vertical and horizontal traffic, as well as a merged dataset, including both activities and behaviors. PAM outperformed LDA in most of the tests with an accuracy of $90.63 \%$ vs. $84.06 \%$ in the vertical traffic dataset, $90.00 \%$ vs. $85.58 \%$ in the horizontal traffic dataset and $87.60 \%$ vs. $83.07 \%$ in the merged dataset. Although MCTM provided the best results in binary behavior classification, this model showed the limitation of the multiclass problem, especial with complex activities comprising two or more phases. Contrary to MCTM, PAM is preferable in the recognition of rare and complex activities due to the captured correlations among the visual words and topics. For future work, the feature extraction algorithm will be considered to increase the processing speed through complexity reduction.

\section{Acknowledgments}

This work was supported by Institute for Information \& communications Technology Promotion (IITP) grant funded by the Korea government (MSIP) (B0101-15-1282-00010002, Suspicious pedestrian tracking using multiple fixed cameras). This work was also supported by the Industrial Core Technology Development Program, funded by the Korean Ministry of Trade, Industry and Energy (MOTIE), under grant number \#10049079. 


\section{Author Contributions}

Thien Huynh-The is the principal researcher of this work. Thien Huynh-The, Ba-Vui Le, Dinh-Mao Bui and Oresti Banos have made significant contributions to the method proposal, experimental evaluation and manuscript preparation. Sungyoung Lee and Yongik Yoon supervised this work. All authors contribute equally to finalizing the manuscript.

\section{Conflicts of Interest}

The authors declare no conflict of interest.

\section{References}

1. Hu, W.; Tan, T.; Wang, L.; Maybank, S. A survey on visual surveillance of object motion and behaviors. IEEE Trans. Syst. Man Cybern. 2004, 34, 334-352.

2. Chaaraoui, A.A.; Climent-Prez, P.; Flrez-Revuelta, F. A review on vision techniques applied to human behaviour analysis for ambientassisted living. Expert Syst. Appl. 2012, 39, 10873-10888.

3. Wiliem, A.; Madasu, V.; Boles, W.; Yarlagadda, P. A suspicious behaviour detection using a context space model for smart surveillance systems. Comput. Vis. Image Und. 2012, 116, 194-209.

4. Brdiczka, O.; Langet, M.; Maisonnasse, J.; Crowley, J.L. Detecting human behavior models from multimodal observation in a smart home. IEEE Trans. Autom. Sci. Eng. 2009, 6, 588-597.

5. Xiang, T.; Gong, S. Beyond tracking: Modelling activity and understanding behaviour. Int. J. Comput. Vis. 2006, 67, 21-51.

6. Kermani, E.; Asemani, D. A robust adaptive algorithm of moving object detection for video surveillance. EURASIP J. Image Video Process. 2014, 2014, doi:10.1186/1687-5281-2014-27.

7. Xiang, T.; Gong, S. Activity based surveillance video content modelling. Pattern Recognit. 2007, 41, 2309-2326.

8. Deepak, N.A.; Hariharan, R.; Sinha, U.N. Cluster based human action recognition using latent dirichlet allocation. In Proceedings of the 2013 International conference on Circuits, Controls and Communications (CCUBE), Bengaluru, India, 27-28 December 2013; pp. 1-4.

9. Modarres, A.F.A.; Soryani, M. Body posture graph: A new graph-based posture descriptor for human behavior recognition. IET Comput. Vis. 2013, 7, 488-499.

10. Zhang, F.; Guo, L.; Lu, H.; Gui, S.; Li, J. Star Skeleton for Human Behavior Recongition. In Proceedings of the 2012 International Conference on Audio, Language and Image Processing (ICALIP), Shanghai, China, 16-18 July 2012; pp. 1046-1050

11. Chakraborty, B.; Rudovic, O.; Gonzalez, J. View-Invariant Human-Body Detection with Extension to Human Action Recognition using Component-Wise HMM of Body Parts. In Proceedings of the 8th IEEE International Conference on Automatic Face \& Gesture Recognition, 2008 (FG'08), Amsterdam, The Netherlands, 17-19 September 2008; pp. 1-6.

12. Duong, T.; Bui, H.; Phung, D.; Venkatesh, S. Activity recognition and abnormality detection with the switching hidden semi-markov model. In Proceedings of the IEEE Computer Vision and Pattern Recognition, San Diego, CA, USA, 20-25 June 2005; pp. 838-845. 
13. Li, C.; Han, Z.; Ye, Q.; Jiao, J. Abnormal behavior detection via Sparse reconstruction analysis of trajectory. In Proceedings of the 6th International Conference on Image and Graphics (ICIG), Hefei, China, 12-15 August 2011; pp. 807-810.

14. Oliver, N.; Rosario, B.; Pentland, A. A bayesian computer vision system for modeling human interactions. IEEE Trans. Pattern Anal. Mach. Intell. 2000, 22, 831-843.

15. Brand, M.; Kettnaker, V. Discovery and segmentation of activities in video. IEEE Trans. Pattern Anal. Mach. Intell. 2000, 22, 844-851.

16. Zhao, L.; Shang, L.; Gao, Y.; Yang, Y.; Jia, X. Video behavior analysis using topic models and rough sets (applications notes). IEEE Comput. Intell. Mag. 2013, 8, 56-67.

17. Chen, Y.; Liang, G.; Lee, K.K.; Xu, Y. Abnormal behavior detection by multi-svm-based bayesian network. In Proceedings of the International Conference on Information Acquisition, Seogwipo-si, Korea, 8-11 July 2007; pp. 298-303.

18. Wang, T.; Snoussi, H. Detection of abnormal events via Optical flow feature analysis. Sensors 2015, 15, 7157-7171.

19. Candamo, J.; Shreve, M.; Goldgof, D.B.; Sapper, D.B.; Kasturi, R. Understanding Transit Scenes: A Survey on Human Behavior-Recognition Algorithms. IEEE Trans. Intell. Transp. Syst. 2009, $11,206-224$.

20. Xiang, T.; Gong, S. Video behavior profiling for anomaly detection. IEEE Trans. Pattern Anal. Mach. Intell. 2008, 30, 893-908.

21. Loy, C.; Xiang, T.; Gong, S. Incremental activity modeling in multiple disjoint cameras. IEEE Trans. Pattern Anal. Mach. Intell. 2012, 34, 1799-1813.

22. Zha, Z.J.; Zhang, H.; Wang, M.; Luan, H.; Chua, T.S. Detecting group activities with multi-camera context. IEEE Trans. Circuits Syst. Video Technol. 2013, 23, 856-869.

23. Huang, B.; Tian, G.; Wu, H.; Zhou, F. A method of abnormal habits recognition in intelligent space. Eng. Appl. Artif. Intell. 2014, 29, 125-133.

24. Medioni, G.; Cohen, I.; Bremond, F.; Hongeng, S.; Nevatia, R. Event Detection and Analysis from Video Streams. IEEE Trans. Pattern Anal. Mach. Intell. 2001, 23, 873-889.

25. Zeng, Y.; Xiang, K. Applying behavior recognition in road detection using vehicle sensor networks. In Proceedings of the International Conference on Computing, Networking and Communication, Maui, HI, USA, 30 January-2 February 2012; pp. 751-755.

26. Sanrom, G.; Patino, L.; Burghouts, G.; Ferryman, J. A unified approach to the recognition of complex actions from sequences of zone-crossings. Img. Vis. Comput. 2014, 32, 363-378.

27. Zhang, Z.; Tan, T.; Huang, K. An extended grammar system for learning and recognizing complex visual events. IEEE Trans. Pattern Anal. Mach. Intell. 2011, 33, 240-255.

28. Hasan, S.; Ukkusuri, S.V. Urban activity pattern classification using topic models from only geo-location data. Trans. Res. Part C 2014, 33, 363-381.

29. Haines, T.; Xiang, T. Delta-dual hierarchical dirichlet processes: A pragmatic abnormal behaviour detector. In Proceedings of the IEEE International Conference on Computer Vision, Barcelona, Spain, 6-13 November 2011; pp. 2198-2205.

30. Hospedales, T.; Gong, S.; Xiang, T. Video behavior mining using a dynamic topic model. Int. J. Comput. Vis. 2011, 98, 303-323. 
31. Wang, X.; Ma, X.; Grimson, W. Unsupervised activity perception in crowded and complicated scenes using hierarchical bayesian models. IEEE Trans. Pattern Anal. Mach. Intell. 2009, 31, $539-555$.

32. Huynh-The, T.; Le, B.; Lee, S.; Yoon, Y.; Le-Tien, T. Using weighted dynamic range for histogram equalization to inprove the image contrast. EURASIP J. Image Video Process. 2014, 2014, doi:10.1186/1687-5281-2014-44.

33. Zhou, H.; Zhang, Z.; Gao, Y.; Yu, P. Video background subtracion using improved adaptive-k gaussian mixture model. In Proceedings of the 3rd International Conference on Advanced Computer Theory and Engineering, Chengdu, China, 20-22 August 2010; pp. 363-366.

34. Barnich, O.; van Droogenbroeck, M. ViBe: A Universal Background Subtraction Algorithm for Video Sequences. IEEE Trans. Image Process. 2011, 20, 1709-1724.

35. Li, W.; McCallum, A. Pachinko allocation dag-structured mixture models of topic correlations. In Proceedings of the 23rd International Conference on Machine Learning, Pittsburgh, PA, USA, 25-29 June 2006; pp. 557-584.

36. Blei, D.; Ng, A.; Jordan, M. Latent Dirichlet allocation. J. Mach. Learn Res. 2003, 17, 993-1022.

37. Li, W.; McCallum, A. Pachinko allocation: Scalable mixture models of topic correlations. J. Mach. Learn. Res. 2008, in press.

38. Cheong, S.; Oh, S.H.; Lee, S.-Y. Support vector machines with binary tree architecture for multi-class classification. Neural Info. Process.-Lett. Rev. 2004, 2, 47-51.

39. QMUL Junction Dataset. Available online: http://www.eecs.qmul.ac.uk/ ccloy/downloads_qmul_ junction.html (accessed on 31-March, 2014 ).

40. Hospedales, T.M.; Li, J.; Gong, S.; Xiang, T. Identifying rare and subtle behaviors: A weakly supervised joint topic model. IEEE Trans. Pattern Anal. Mach. Intell. 2011, 33, 2451-2464.

(c) 2015 by the authors; licensee MDPI, Basel, Switzerland. This article is an open access article distributed under the terms and conditions of the Creative Commons Attribution license (http://creativecommons.org/licenses/by/4.0/). 